\title{
A COMPARATIVE STUDY BETWEEN AGRICULTURAL LEADERS AND FARMERS IN THE FIELD OF ADVANCEMENT AND MAINTENANCE OF RECLAIMED LAND AT KAFR EL-SHAIKH GOVERNORATE
}

Shalaby, Ebtesam H. and E.S. Mikhael

Agric. Ext. and Rural development Research Institute

دراسة مقارنة بين القيادات الززراعية والزراع فى مجال تحسين وصيحــــة التربــة بالأراضى الجديدة بمحافظة كفر الشيخ الشيخ ابتسام حامد شُببى وإميل صبحى ميذاتيليل معه بحرث الإرثياد الزراعى والتعية الريفية

\section{الملخص}

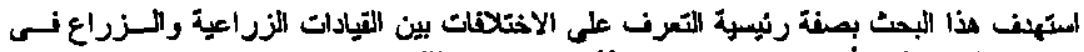

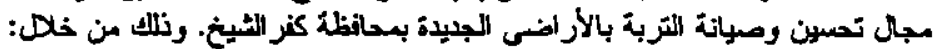

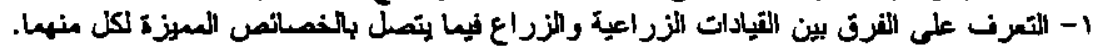

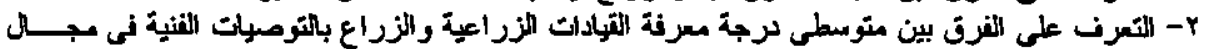
تحسين وصياتة التربة.

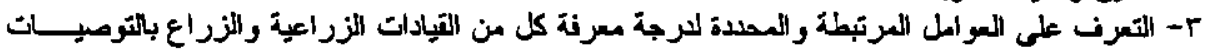
الالنية نى مجال تحسين وصباتة التربة.

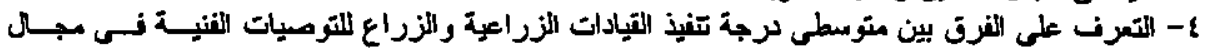
تصسين وصبانة التزبة.

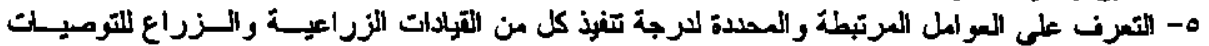

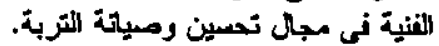

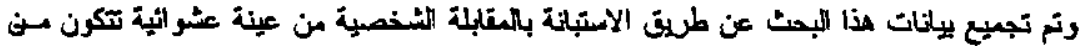

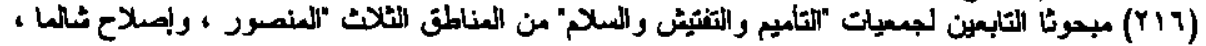

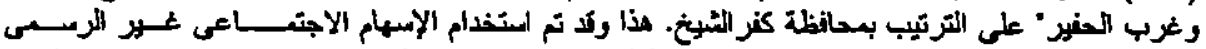

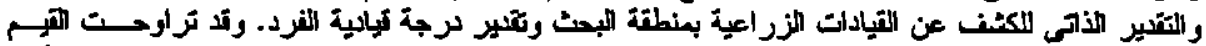

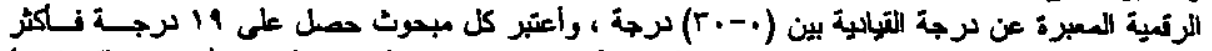

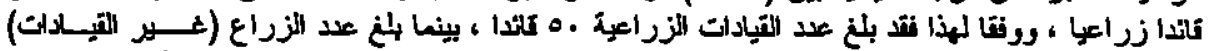

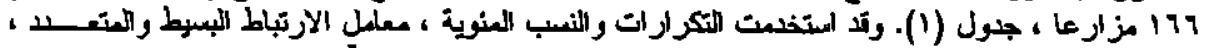

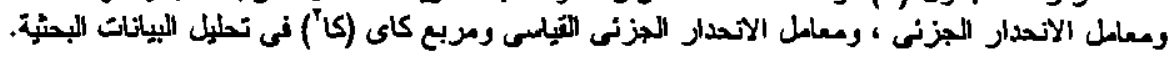

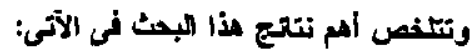

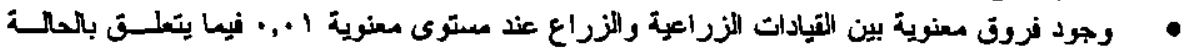

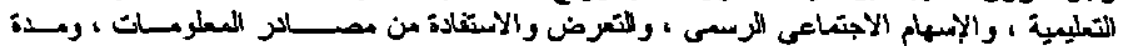

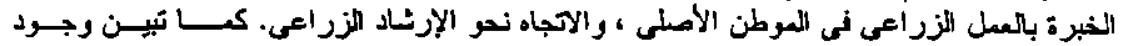

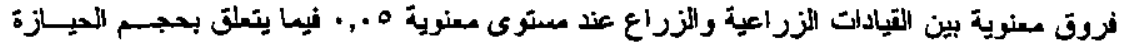

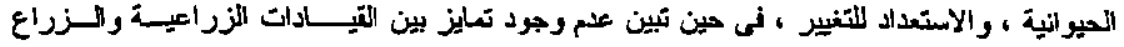
بالنعبه لباتى الخمائص.

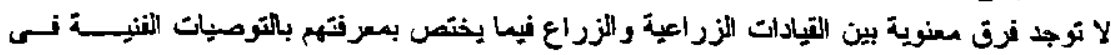
مجال تحسن وصياتة الثربة. 


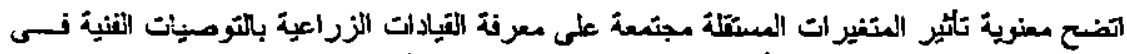

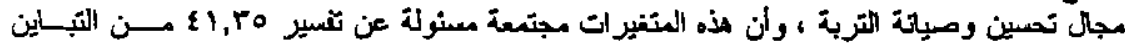

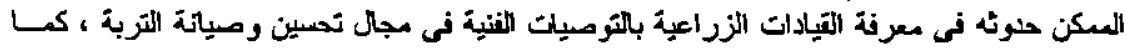

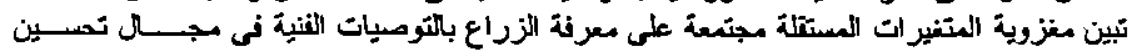

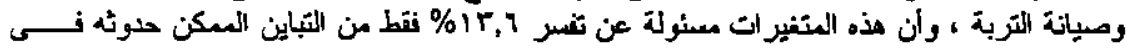

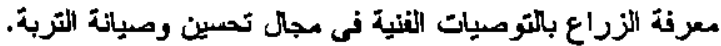

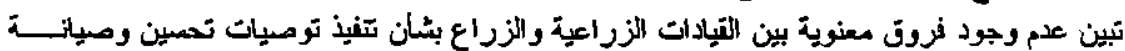
التزبة.

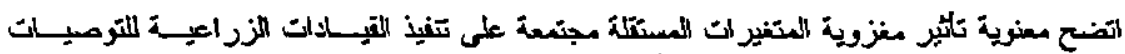

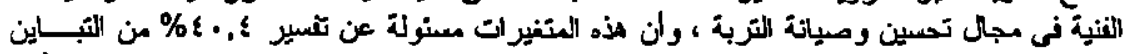

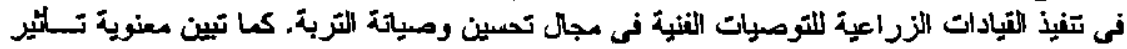

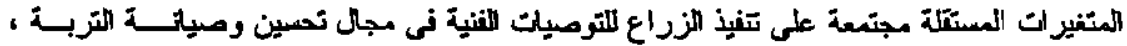

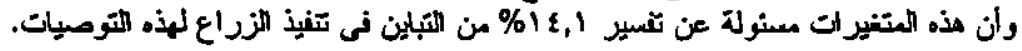

\section{المقديمـة}

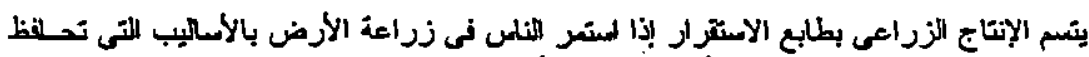

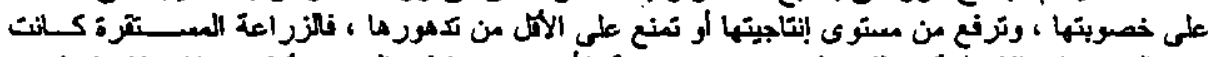

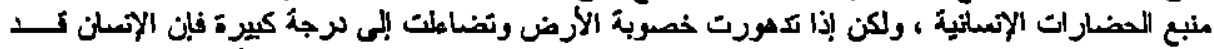

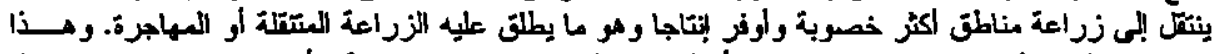

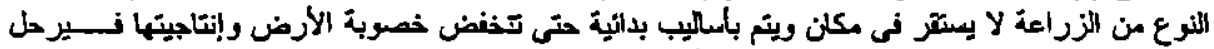

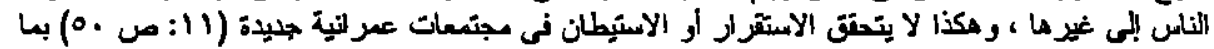

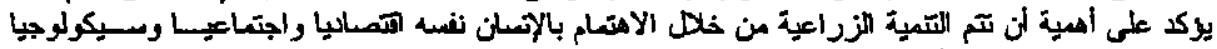

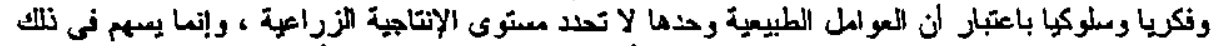

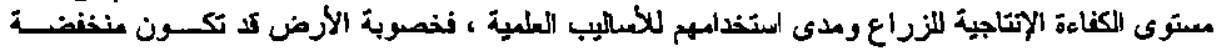

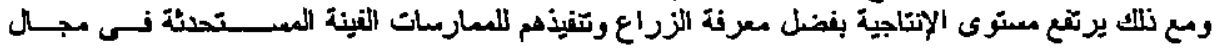
تحسين وصيانة التزبة والعكس صحيح.

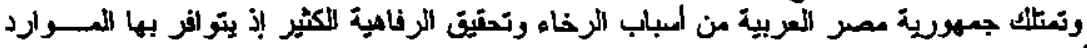

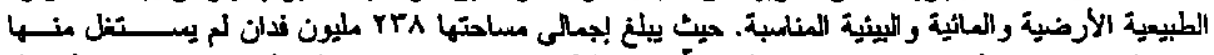

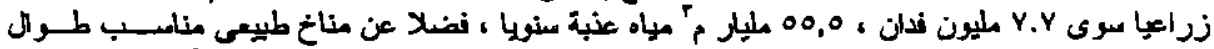

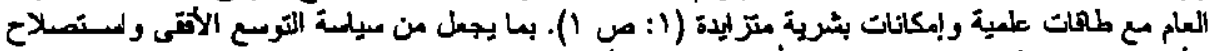

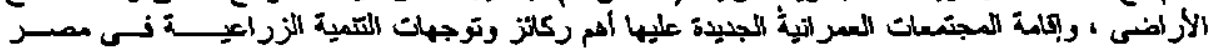

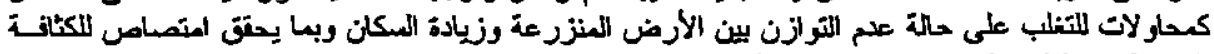

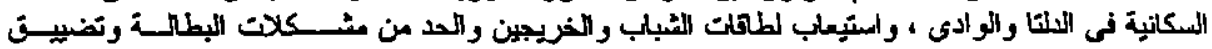

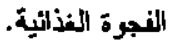

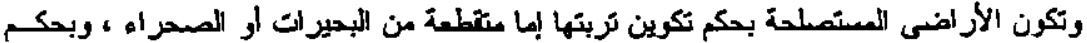

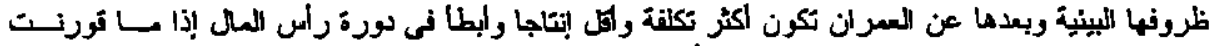

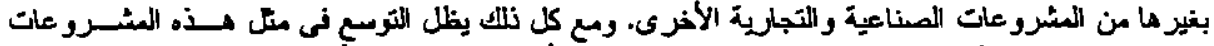

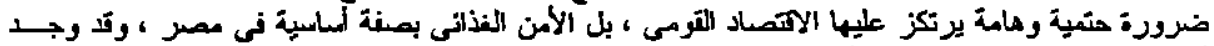

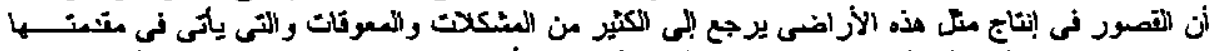

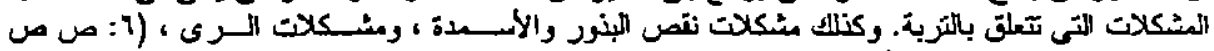

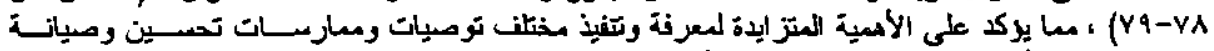

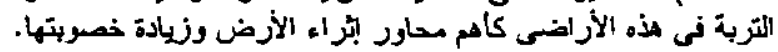

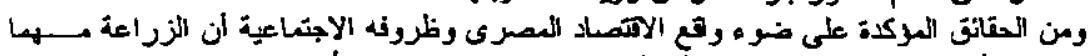

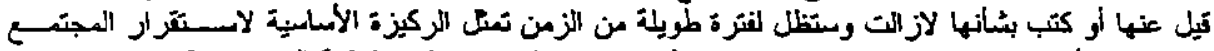

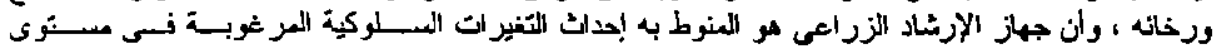




\section{J. Agric. Scl. Mansoura Univ., 29(5), May, 2004}

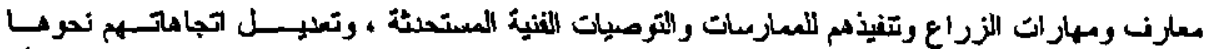

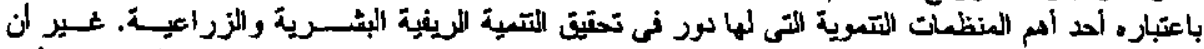

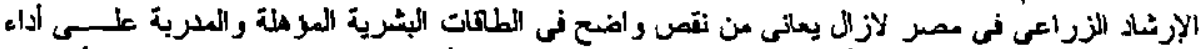

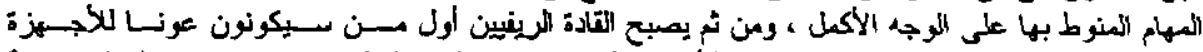

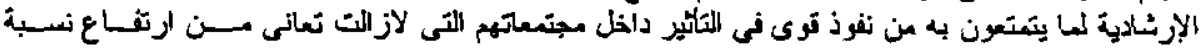

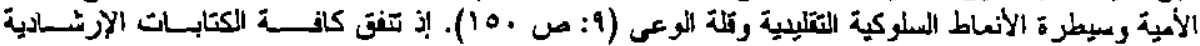

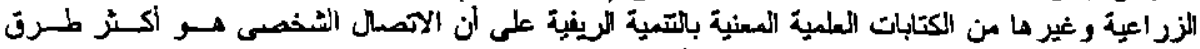

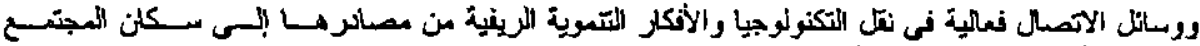

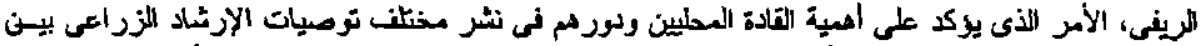

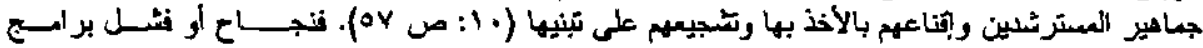

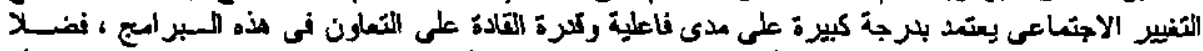

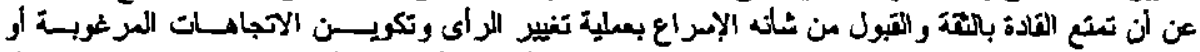

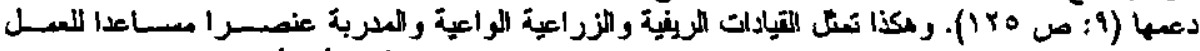

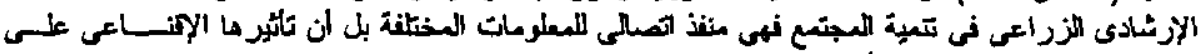

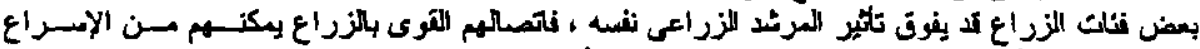

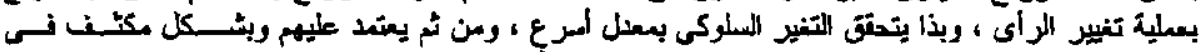

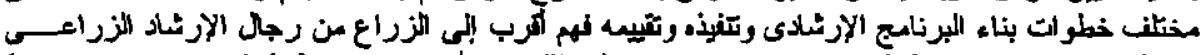

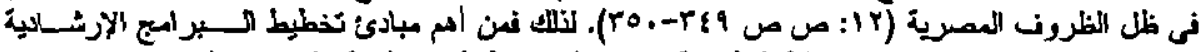

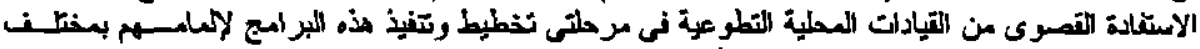

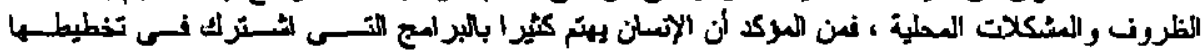

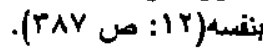

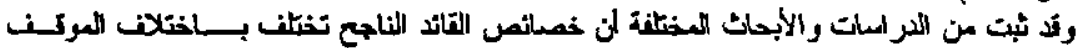

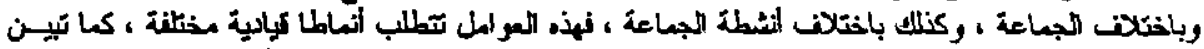

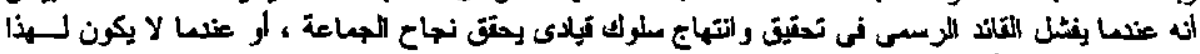

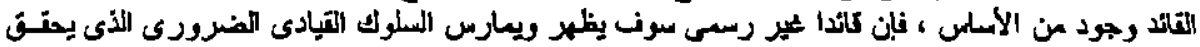

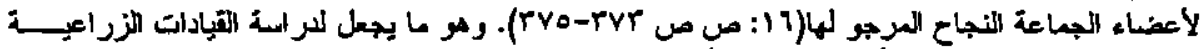

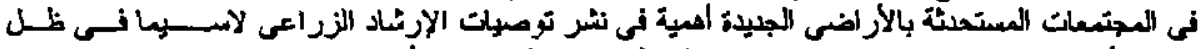

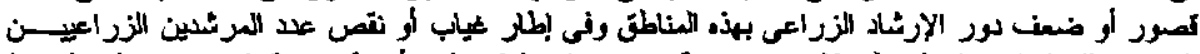

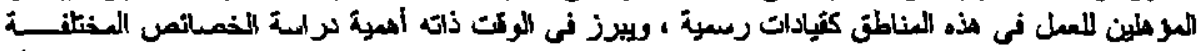

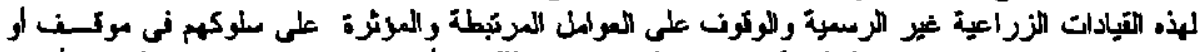

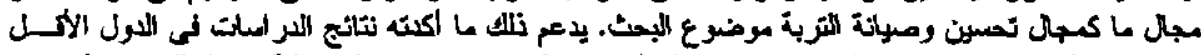

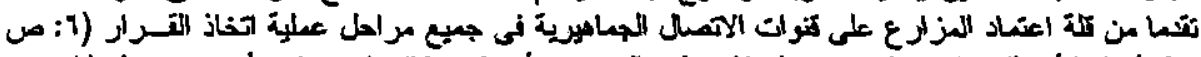

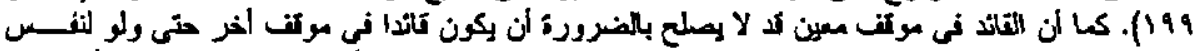

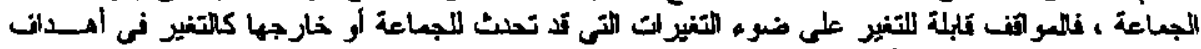

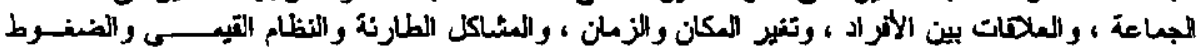

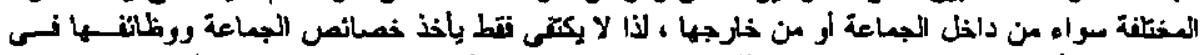

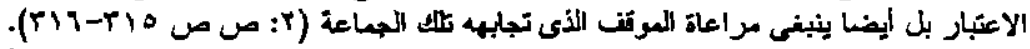

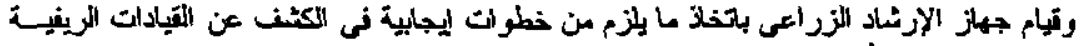

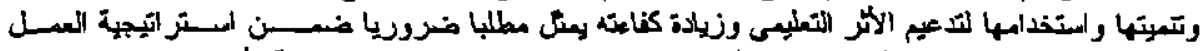

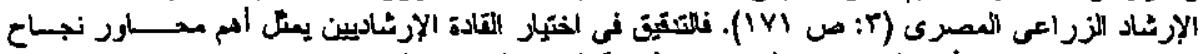

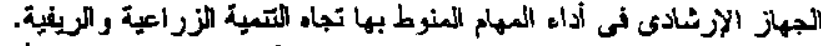

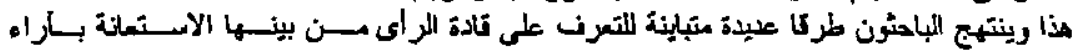

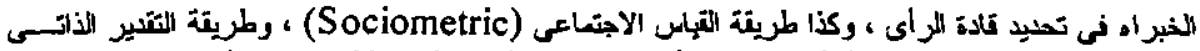

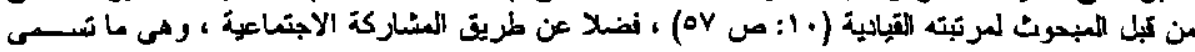

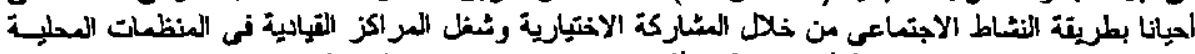

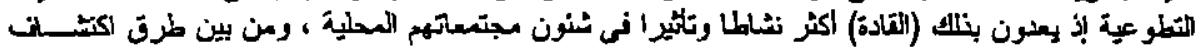




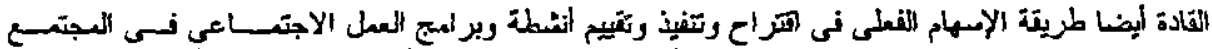

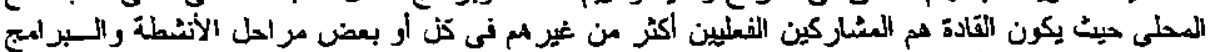

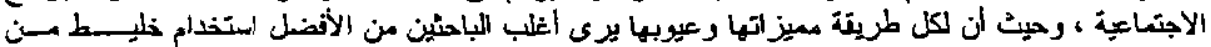

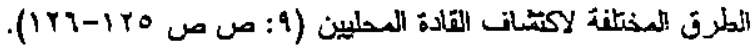

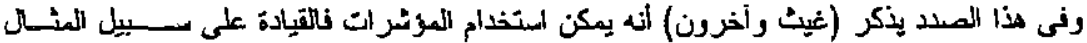

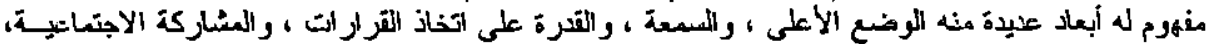

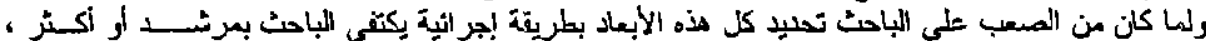

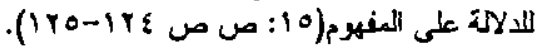

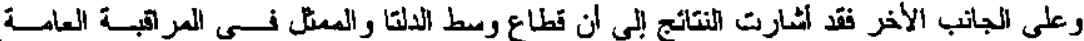

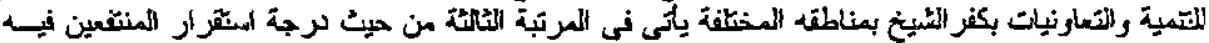

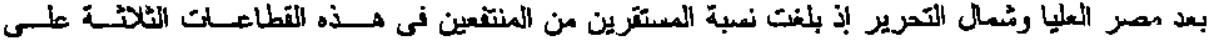

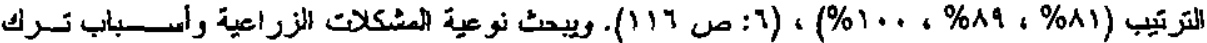

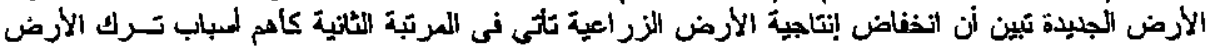

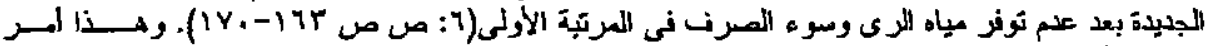

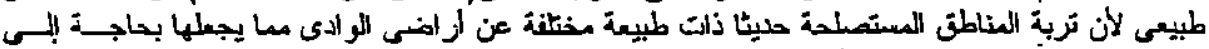

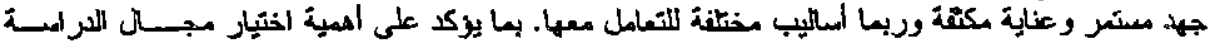

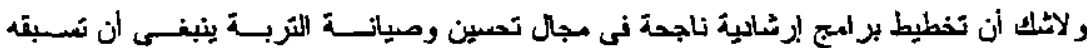
ومنطتة البحثث.

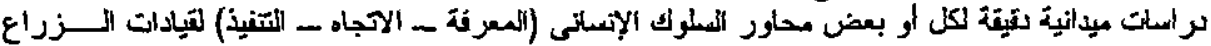

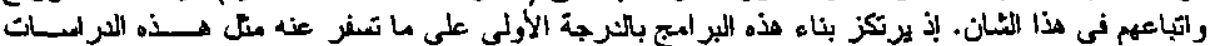

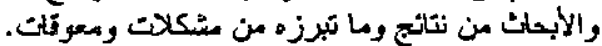

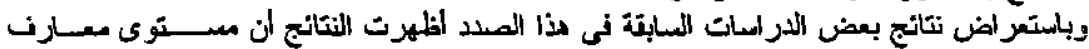

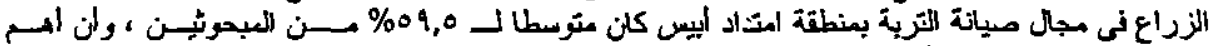

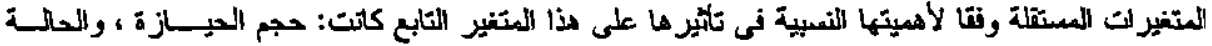

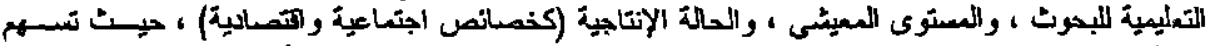

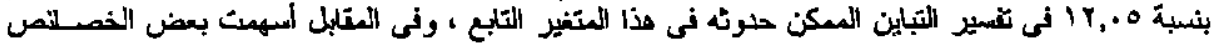

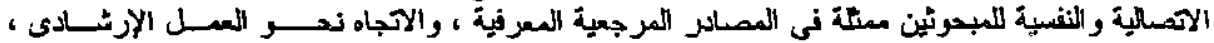

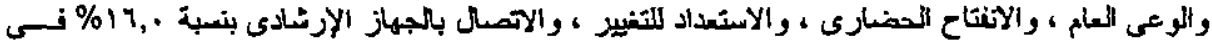

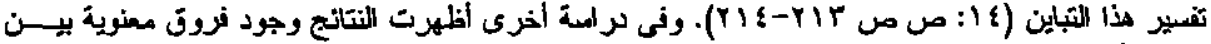

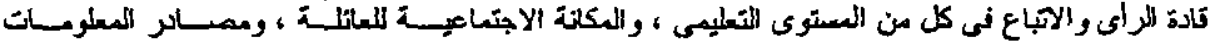

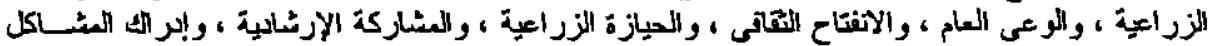

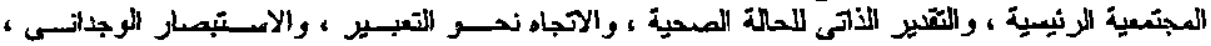

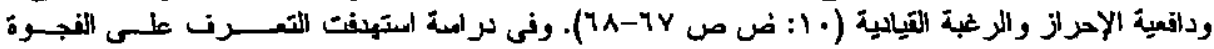

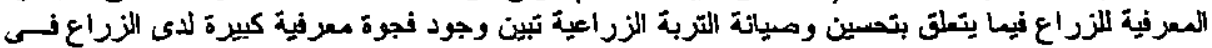

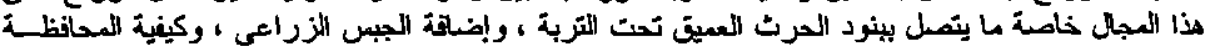

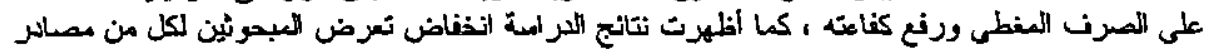

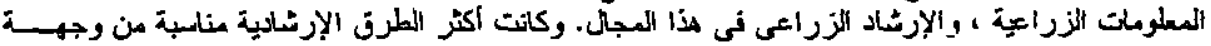

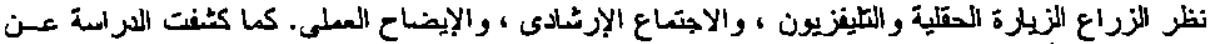

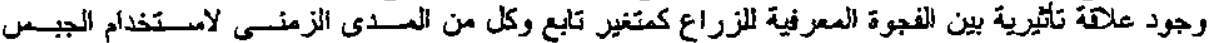

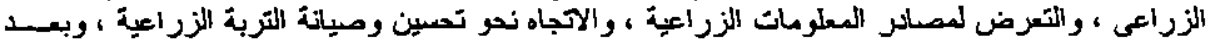

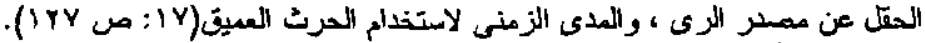

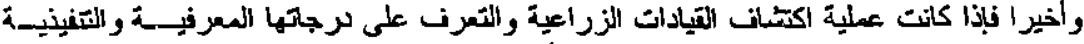

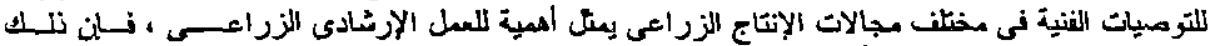

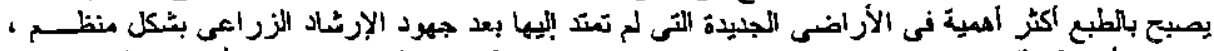

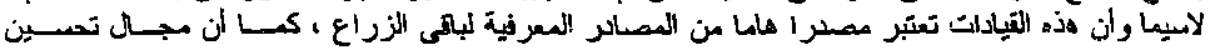

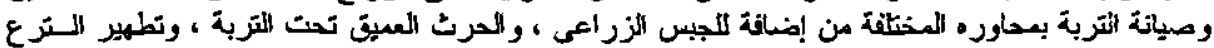




\section{J. Agric. Scl. Mansoura UnIv., 29(5), May, 2004}

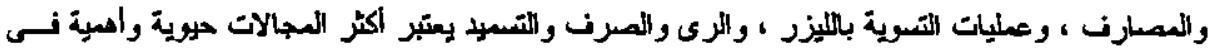

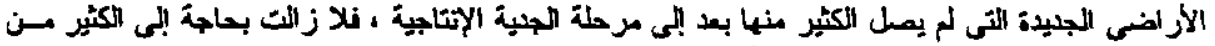

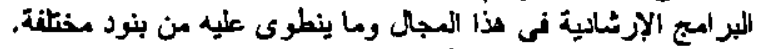

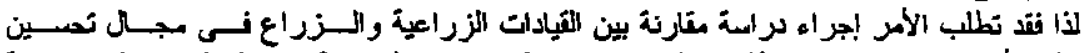

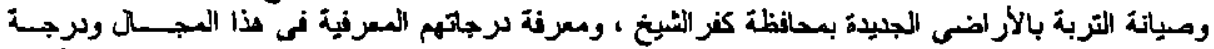

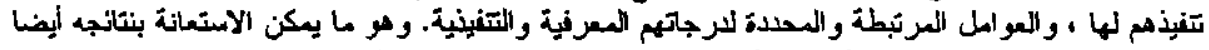

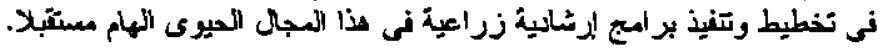

\section{أن إن}

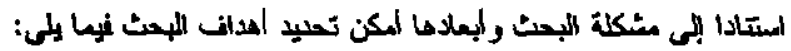

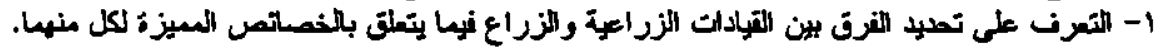

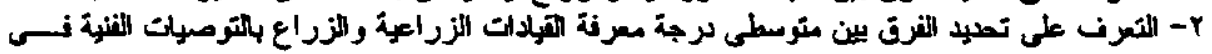
مجل تعمين وميلة الترية.

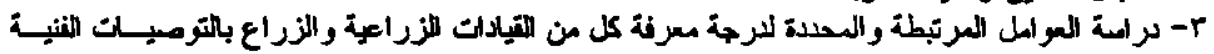
فمى مجال تعسين وصياتة التربة.

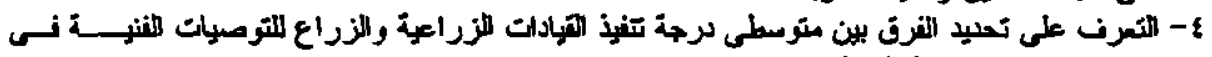
مجل تحبين وصباتة التربة.

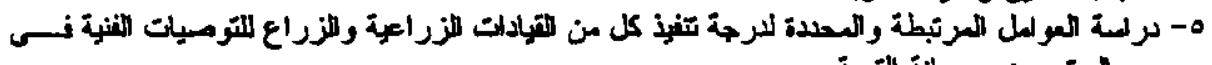
مجل تصعين وصبانة التزبة.

$$
\text { الأسلوب البحثى }
$$

المامدم الإجراثة:

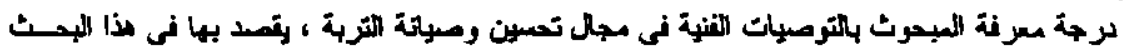

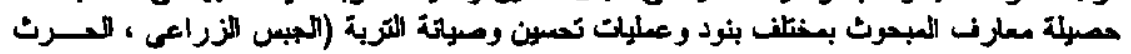

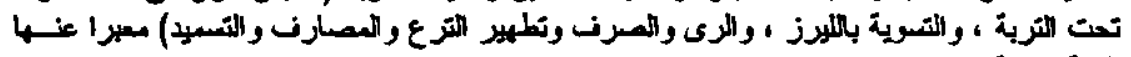

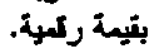

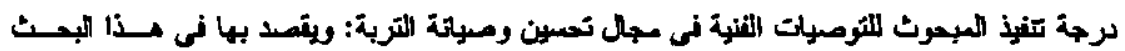

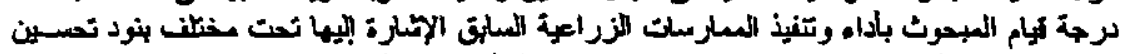

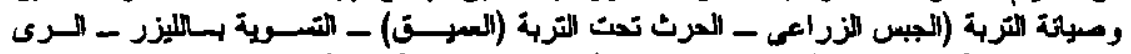

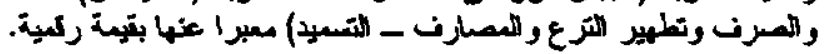

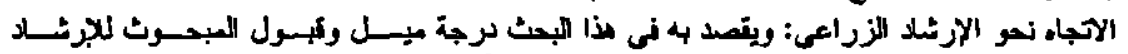

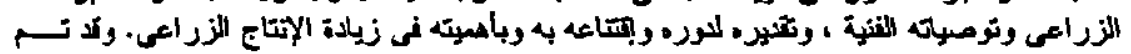

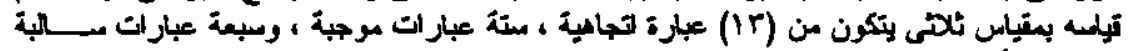

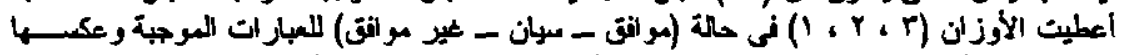

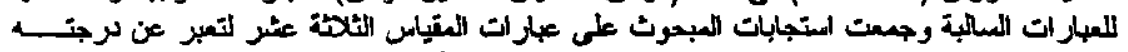

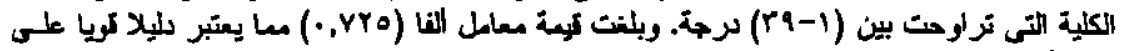

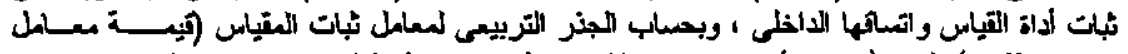

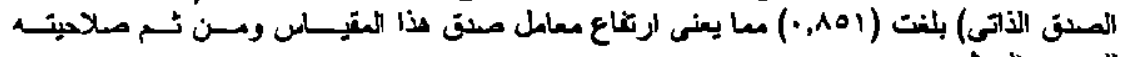

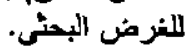

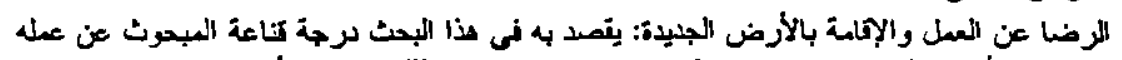

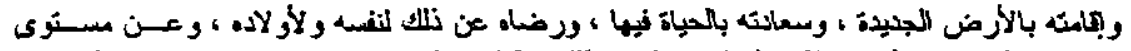

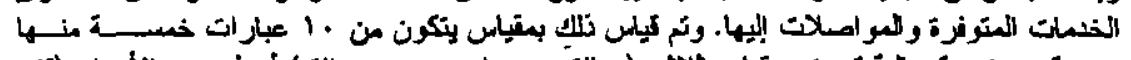

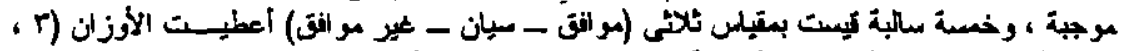

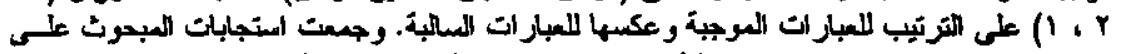

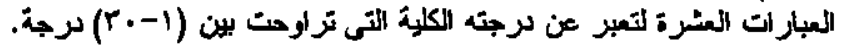




\section{Shalaby, Ebtesam H. and E.S. Mlkhael}

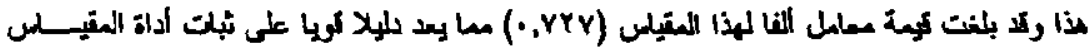

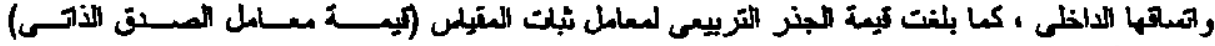

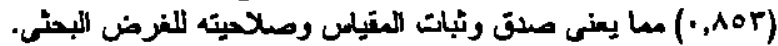

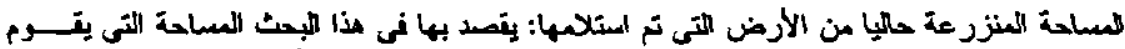

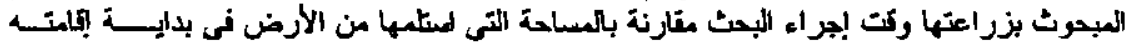

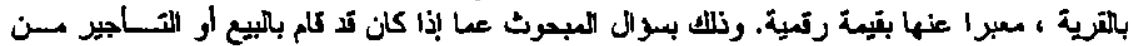

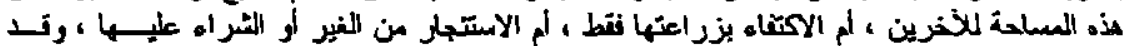

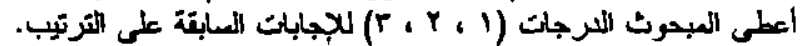

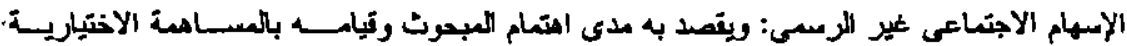

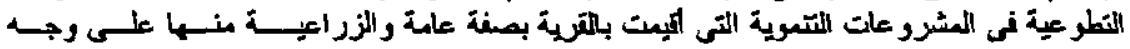

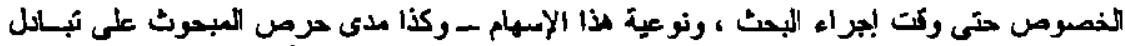

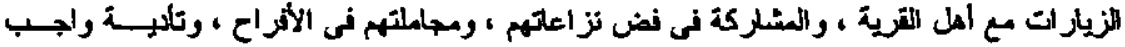

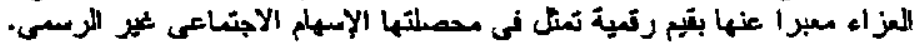

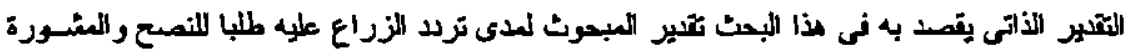

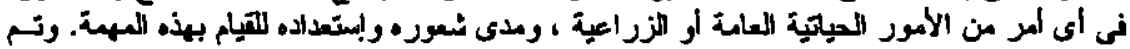

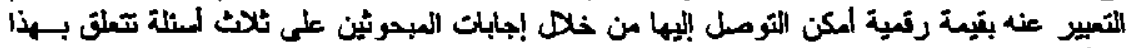
اللطان.

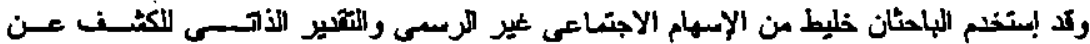

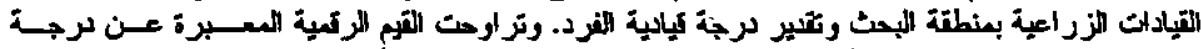

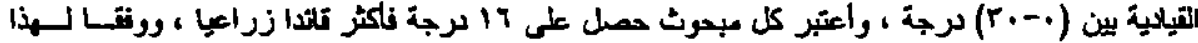

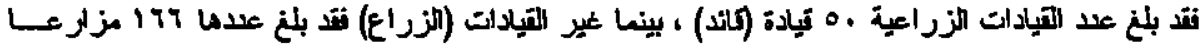

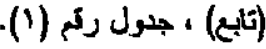

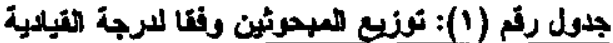

\begin{tabular}{|c|c|c|}
\hline$\%$ & العثد & درجة الترئية \\
\hline$V i, 10$ & 177 & الززراع (صفز - 10 درجة) \\
\hline$Y r, 10$ & 0. & القِياداتث الزراعية (14 - ·r درجة) \\
\hline $1 \ldots$ & Y14 & العجموع \\
\hline
\end{tabular}

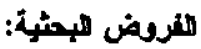

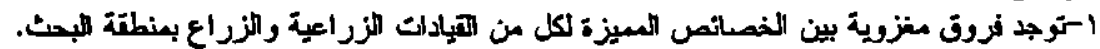

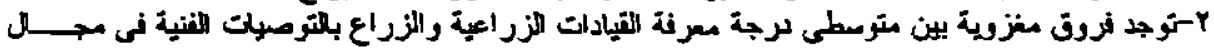

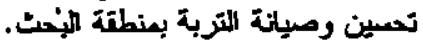

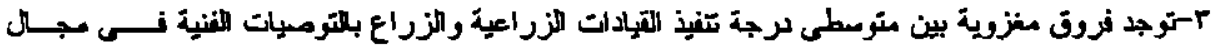

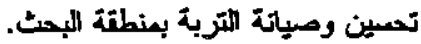

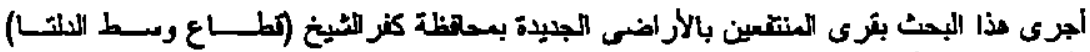

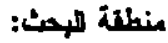

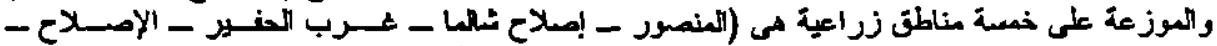

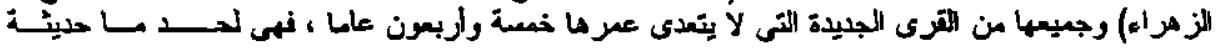

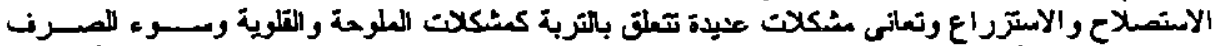

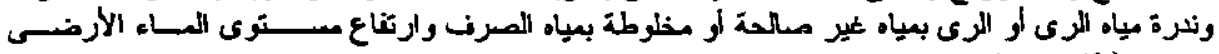
ورجود طبقات صناء.

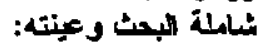

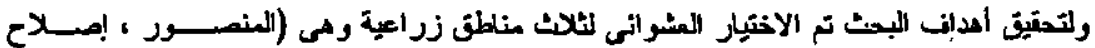

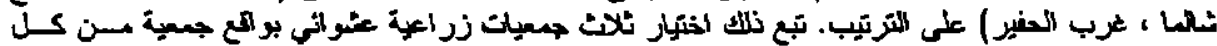

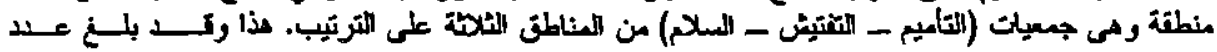




\section{J. Agric. Scl. Mansoura Univ., 29(5), May, 2004}

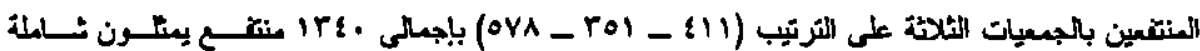
البعث.

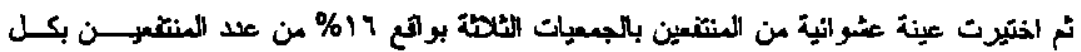

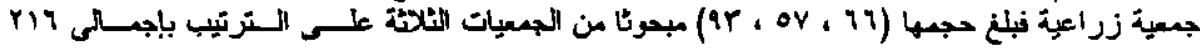
مبحوث.

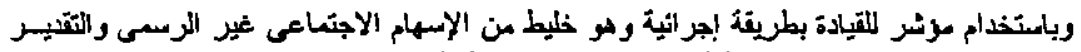

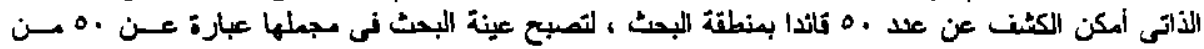

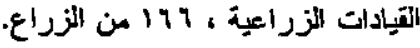

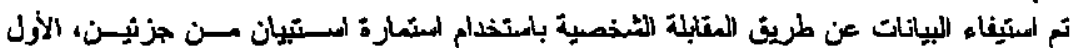
جمع بوحات البحث:

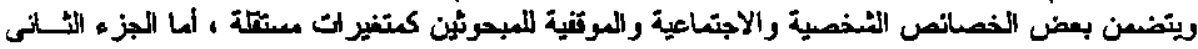

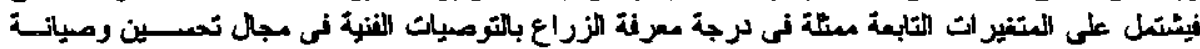

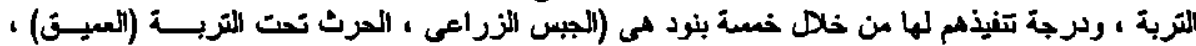

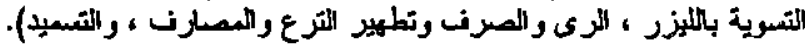

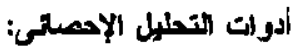

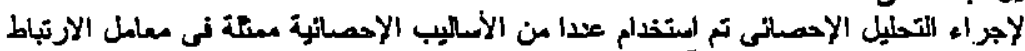

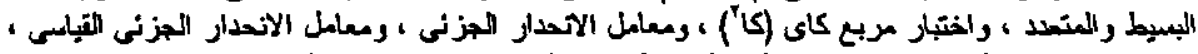

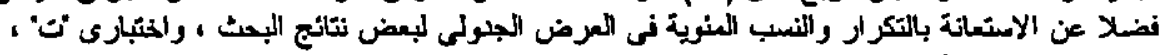

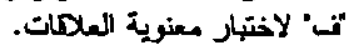

\section{النتأتع وناتشتها}

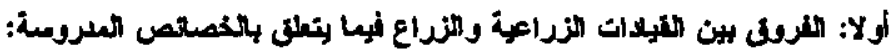

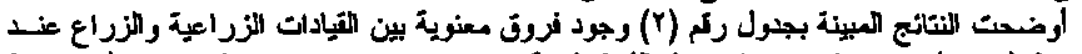

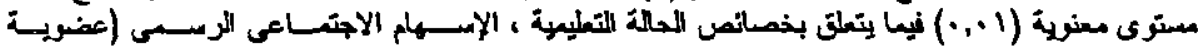

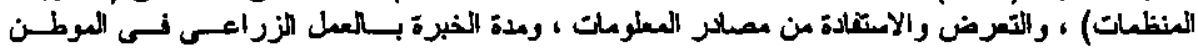

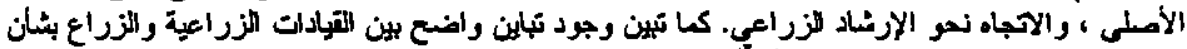

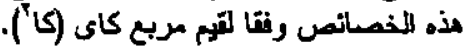

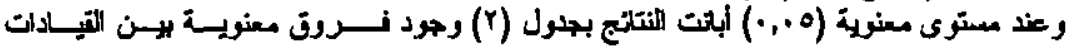

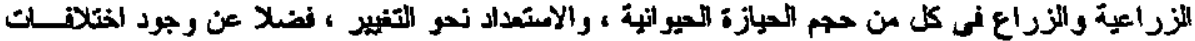

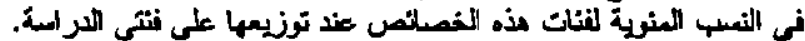

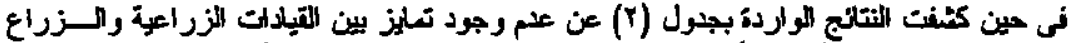

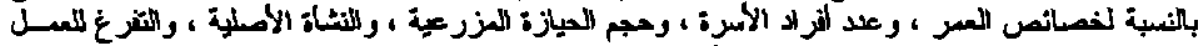

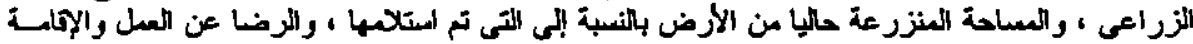

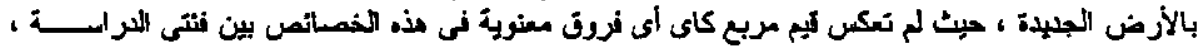

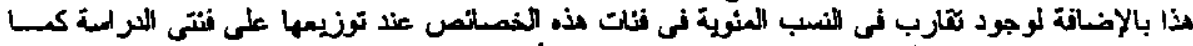

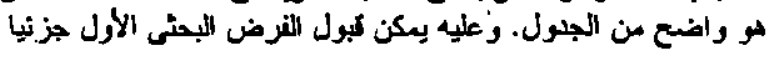

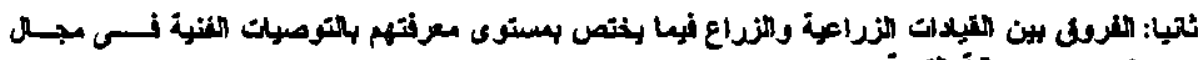
تصعين وصرية التربة:

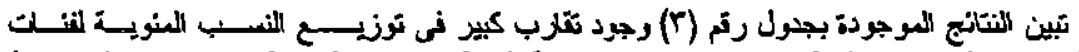

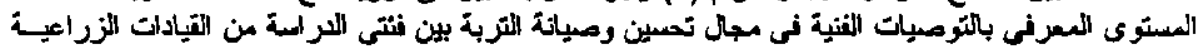

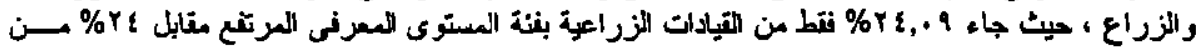

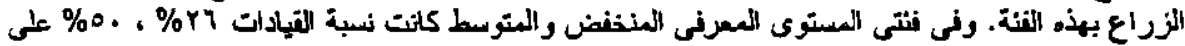

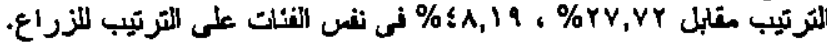


Shalaby, Ebtesam H. and E.S. MIkhael

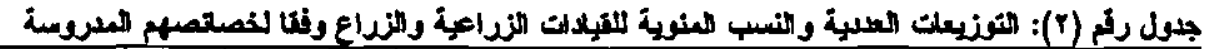

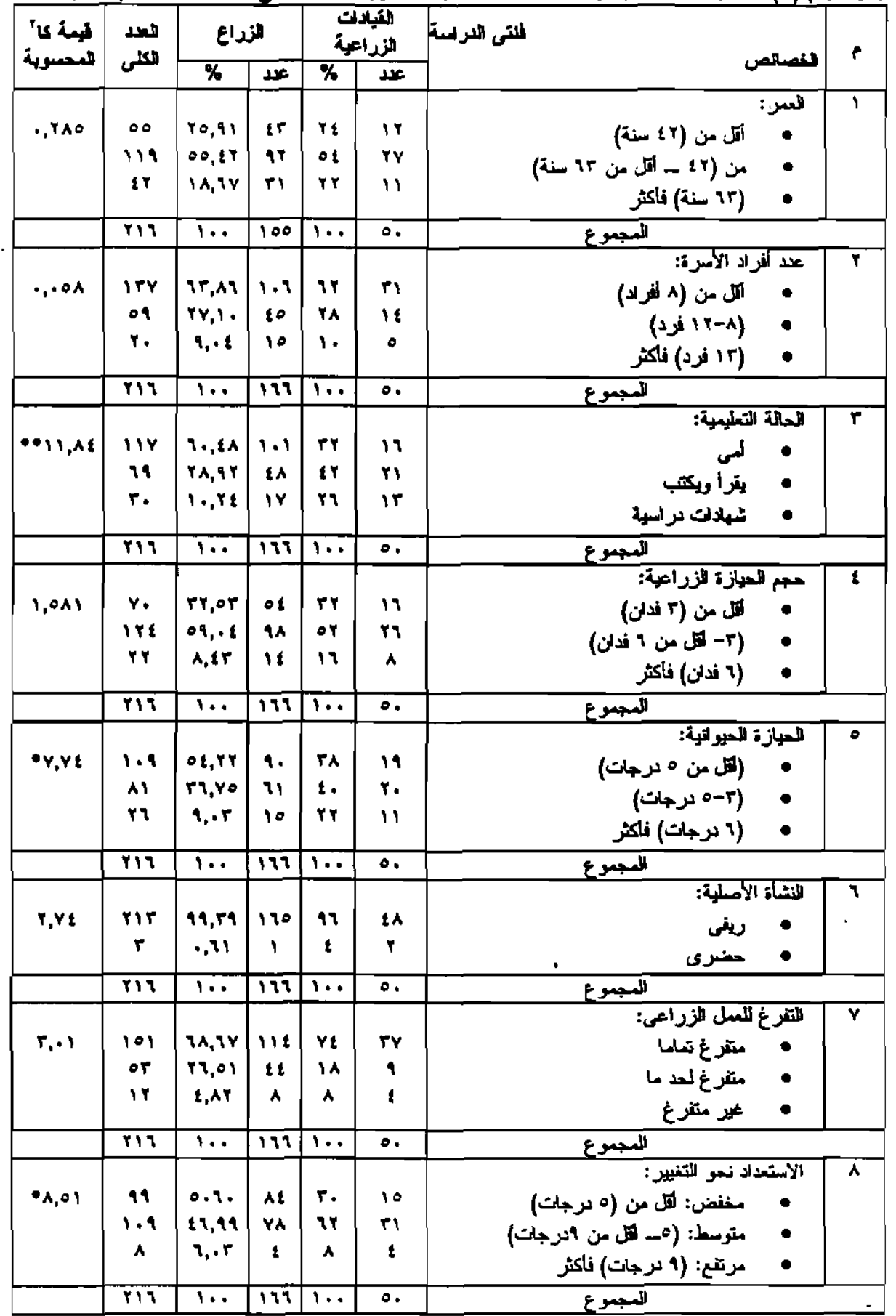


J. Agric. Scl. Mansoura Univ., 29(5), May, 2004

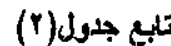

\begin{tabular}{|c|c|c|c|c|c|c|c|}
\hline 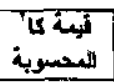 & الكئد & \multicolumn{2}{|c|}{ المزداع } & \multicolumn{2}{|c|}{ 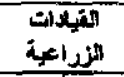 } & 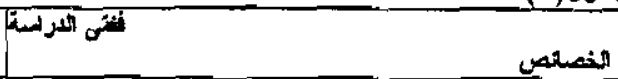 & م \\
\hline$\cdots, r, r r$ & $\begin{array}{l}\text { ind } \\
11 \\
\text { iv }\end{array}$ & $\begin{array}{l}91,0 y \\
r,+1 \\
0,2 r\end{array}$ & $\begin{array}{l}10 \% \\
0 \\
4\end{array}$ & $\begin{array}{l}V Y \\
17 \\
17\end{array}$ & $\begin{array}{l}4 \\
1 \\
1\end{array}$ & 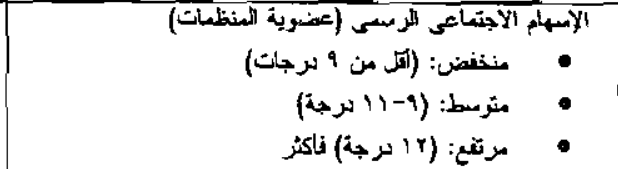 & 9 \\
\hline & 718 & $\cdots$ & 177 & $1 \ldots$ & o. & المجسوع & \\
\hline$\cdots 13, r !$ & $\begin{array}{l}\text { iro } \\
\text { VT } \\
\text { IA }\end{array}$ & $\begin{array}{l}r, A s \\
r i, r o \\
l, \lambda r\end{array}$ & $\begin{array}{l}1.9 \\
\text { or } \\
1\end{array}$ & $\begin{array}{l}T \lambda \\
q r \\
Y .\end{array}$ & $\begin{array}{l}19 \\
11 \\
1 .\end{array}$ & 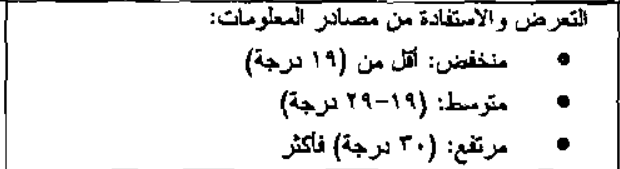 & 1. \\
\hline & Y14 & $\cdots$ & 179 & $\cdots$ & 0. & المجبموع & \\
\hline $4, \cdots$ & $\begin{array}{l}\text { Tr } \\
104 \\
\text { To }\end{array}$ & $\begin{array}{l}1 \cdot, A \\
Y \varepsilon, Y \\
1 \&, 8\end{array}$ & $\begin{array}{l}14 \\
17 q \\
r q\end{array}$ & $\begin{array}{l}\text { in } \\
\text { ra } \\
\text { YY }\end{array}$ & $\begin{array}{l}9 \\
\text { r. } \\
11\end{array}$ & 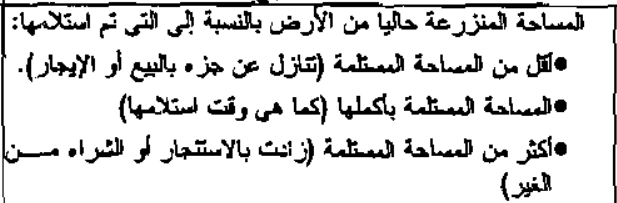 & 11 \\
\hline & Y17 & $1 \ldots$ & 179 & $1 \cdots$ & $\therefore$ & المجسوع & \\
\hline$\cdot \operatorname{lo,rr}$ & $\begin{array}{l}Y_{0} \\
11 E \\
\text { YY }\end{array}$ & $\begin{array}{l}Y Y, Y \mid \\
0 Q, \cdot Y Z \\
\mid r, Y O\end{array}$ & $\begin{array}{l}\{4 \\
9 A \\
Y Y\end{array}$ & $\begin{array}{l}O A \\
r Y \\
1 .\end{array}$ & $\begin{array}{l}81 \\
17 \\
0\end{array}$ & 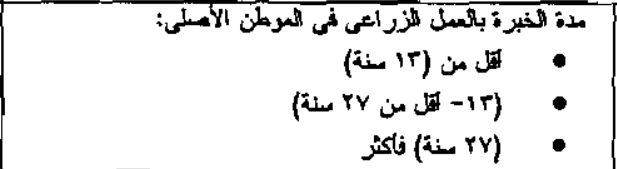 & iY \\
\hline & 817 & $1 \cdots$ & 174 & $1 \ldots$ & 0. & المجمرع & \\
\hline - $|r . A|$ & $\begin{array}{c}Y Y \\
\text { ir } \\
\text { IrY }\end{array}$ & $\begin{array}{l}r, \cdot T \\
r T, 1 T \\
r ., A \varepsilon\end{array}$ & $\begin{array}{l}1 . \\
80 \\
1.1\end{array}$ & $\begin{array}{l}Y ! \\
r t \\
\text { Or }\end{array}$ & $\begin{array}{l}\text { ir } \\
\text { ir } \\
\text { ry }\end{array}$ & 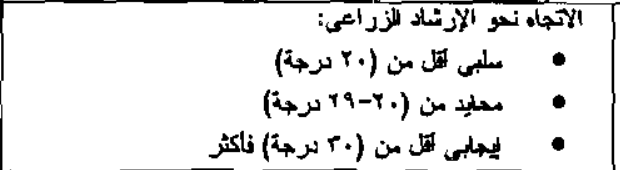 & TT \\
\hline & 917 & 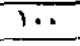 & 177 & $\cdots$ & 0. & الل المجموع & \\
\hline$\{, Y 0$ & $\begin{array}{l}\text { An } \\
\text { it } \\
\text { is }\end{array}$ & $\begin{array}{l}Y 9, \Delta Y \\
r 9,1\} \\
T 1, r Y\end{array}$ & $\begin{array}{l}\text { iq } \\
\text { is } \\
\text { or }\end{array}$ & $\begin{array}{l}11 \\
87 \\
74\end{array}$ & $\begin{array}{l}q \\
\text { YA } \\
\text { it }\end{array}$ & 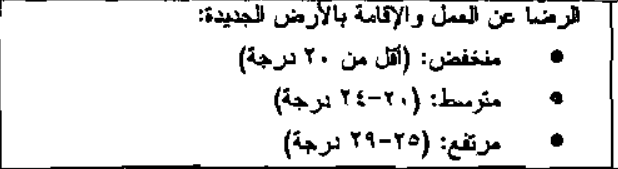 & $1 \xi$ \\
\hline & 817 & $\cdots$ & 178 & $1 \ldots$ & $\therefore$ & العجموع & \\
\hline
\end{tabular}

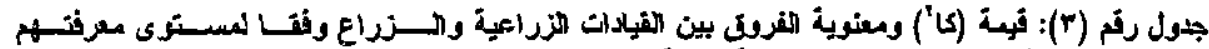

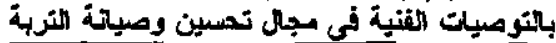

\begin{tabular}{|c|c|c|c|c|c|c|c|}
\hline قيمة كا" & المعدد & $\varepsilon !$ & & زراتية & 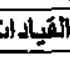 & ل العرفة بالتوصيات الفنية & مستو \\
\hline |المحسوبة & الإجمالى & $\%$ & عدد & $\%$ & عدد & & \\
\hline & 09 & $\overline{Y Y, Y Y}$ & 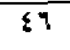 & Y7 & 15 & عنففض: أقل من (rT برجة) & $\bullet$ \\
\hline$\cdot, \cdot Y$ & 1.0 & $\{\wedge, \uparrow \uparrow$ & $\lambda$. & $\circ$. & ro & 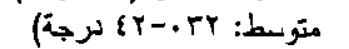 & - \\
\hline & or & $r \varepsilon_{1}, q$ & $\varepsilon$. & $Y \varepsilon$ & ir & مزتفع: (ז؛ نرجة) فاكثر & - \\
\hline & 819 & $T \ldots$ & 179 & $1 \cdots$ & 0. & اللمجموع & \\
\hline
\end{tabular}




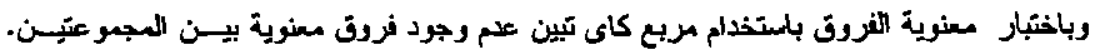

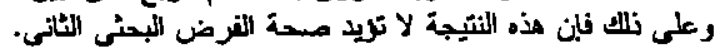

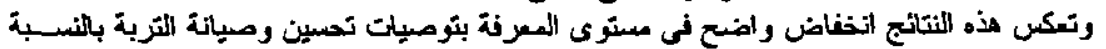

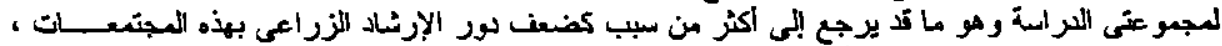

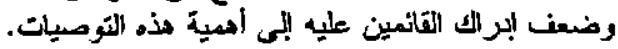

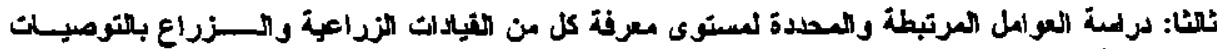

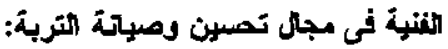

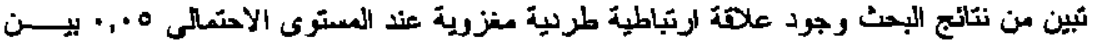

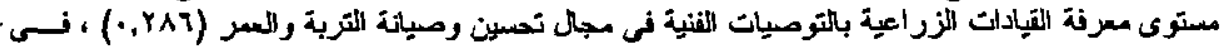

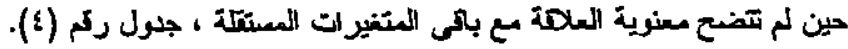

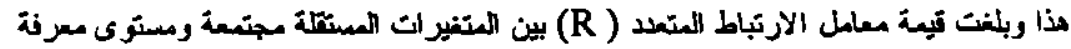

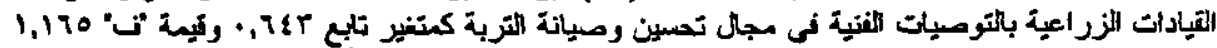

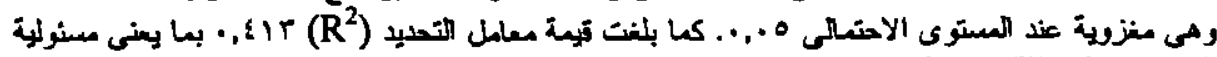

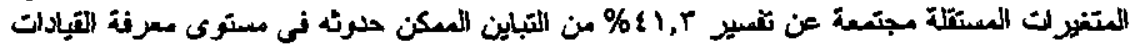

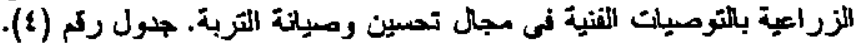

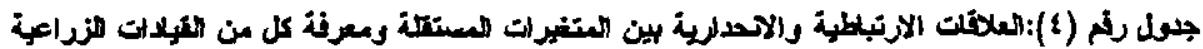

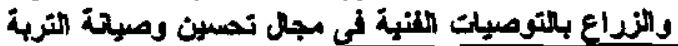

\begin{tabular}{|c|c|c|c|c|c|c|c|c|}
\hline \multicolumn{4}{|c|}{ هزراع } & \multicolumn{4}{|c|}{ الفبلات التزداعة } & \multirow{2}{*}{ 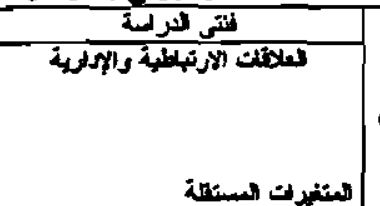 } \\
\hline "انى" & 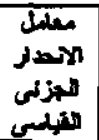 & "يلمطنم & 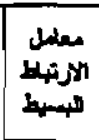 & "الهة & $\mid$ & "لآططالر & 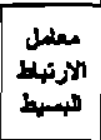 & \\
\hline T.rAn & $.1 \% \mathrm{t}$ & $y, 20$. & $\cdot \because \cdot \lambda \lambda$ & - $1,4 \mathrm{tYV}$ &., 0.1 & $\cdot$, rOA & $\because, Y \Lambda T$ & أقعر لَبحوث \\
\hline 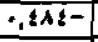 & $\cdot . \cdot 4 \cdot-$ & $A_{.}[1 A-$ & $\because .9 Y$ & $1,2 \wedge\{-$ & $\because \mathrm{YYO}-$ & $., 08,-$ &., $109-$ & 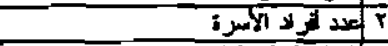 \\
\hline$\therefore$, AOr & $\because \because \mathrm{YA}$ &., 190 & $\cdot . \cdot 97$ & $\because \cdot 0,1 \%$. & $\cdot, 7+7$ & $.9 \mathrm{AY}$ & $\because, \Gamma_{\Lambda}$ & بالمالقة كتمليمية المبحوث \\
\hline$\because .19$ & $\cdots 1$ & $P, 1 ! 4$ & ?.01r- &., 11 & 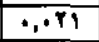 & $\overline{Y, O A O}$ & $\cdot, \cdot A Y$ & \& \\
\hline $1,1.9$ & $\because \bullet \mathrm{AA}$ & $\cdot, \Gamma, r$ & $\cdot ., .79$ & . VAT &., 11 & . Yar & 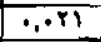 & 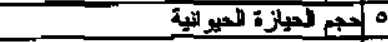 \\
\hline$\cdot .1 \mathrm{rr}-$ & $\because .011-$ & $T, 7 A^{-}$ & $\because .91$ & $\cdot, 81 .-$ & $\because \cdot v_{t}-$ & .,YTY- & $\cdot, 9$. & 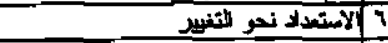 \\
\hline 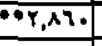 & $. . \mathrm{Y} \leqslant 5$ & .0.0\% & $\because$. por & $1, Y 9 \pi$ &., 199 & $\cdot, \mathrm{YTT}$ &.,$Y i Y$ & 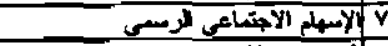 \\
\hline$\cdot, \mathrm{rrT}-$ & $\cdot$, . 1 - & $1, r r-$ & $\cdot ., 18$ &.,$r r i$ & 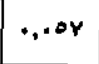 & $0,08 Y$ & $\because .180-$ & 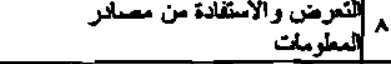 \\
\hline $1, \mathrm{rA}-$ & $\cdot, 1 \cdot 0-$ & $1,\{11-$ & -.117- & $1, n / \gamma-$ & - & $r, \cdot 1-$ & $-117-$ & 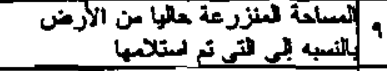 \\
\hline •.หาר- & $\cdot, \cdot 1-$ & $r, q r-$ & •, $\times \&-$ & $1, \mathrm{ruV}$ & $\because Y \wedge A$ & $\cdot|A|$ & $\cdot .1 T$ & 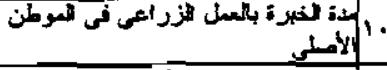 \\
\hline., $700-$ & $\because .00 \mathrm{AN}$ & $\lambda_{1} \cdot-$ &.,$\cdot \square !-$ & $1, V T A-$ & . rist- &., $0 Y A^{-}$ & $\therefore, r \leqslant 9-$ & 1الاتجاء نحو الارثماد الزراعم \\
\hline $\bar{r}$ & $\because Y \cdot A-$ & $\cdot, \mathrm{r} 20-$ & & 109- & $A_{i}-$ & $\cdot$, raq- & $\cdot, 1 \cdot\{-$ & 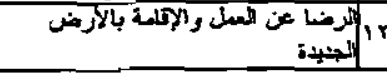 \\
\hline & & R) & b & & & & & 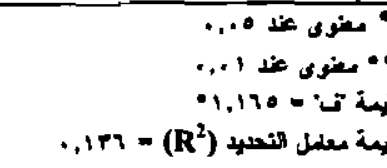 \\
\hline
\end{tabular}

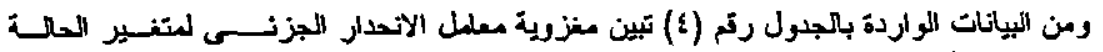

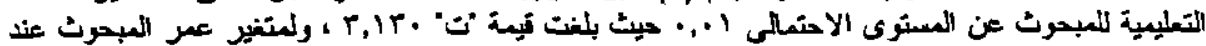

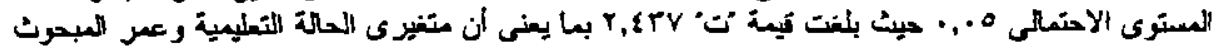

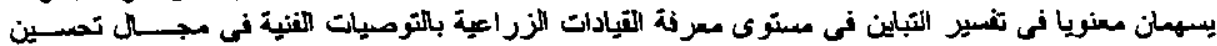
وصياتة التربة. 


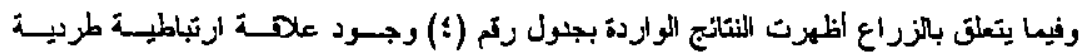

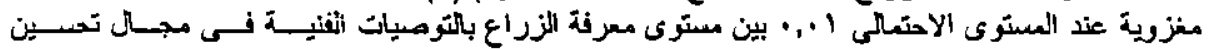

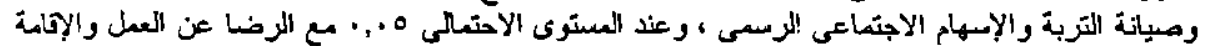

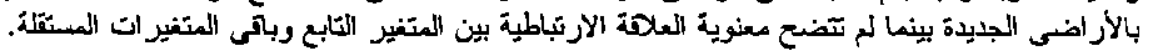

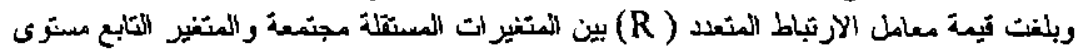

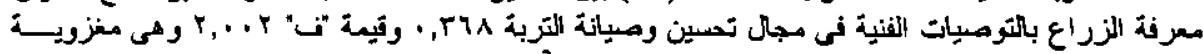

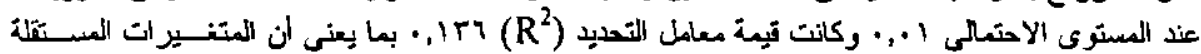

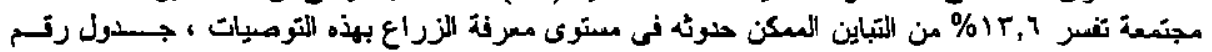

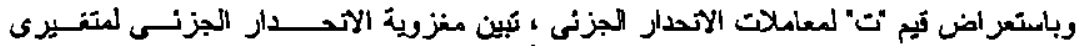

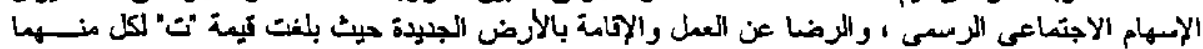

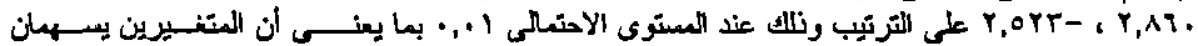

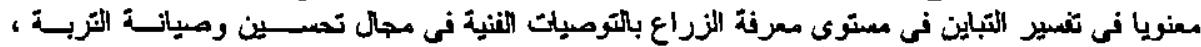

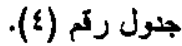

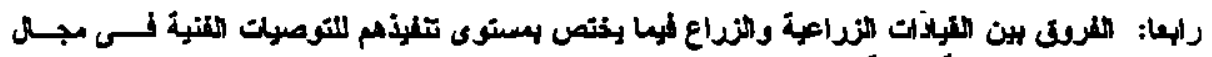
تصني وصبحة التربة:

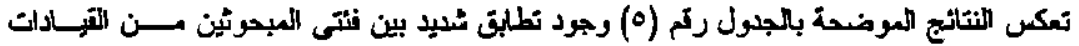

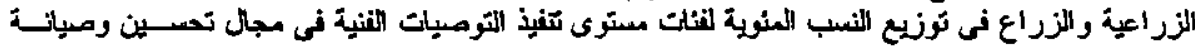

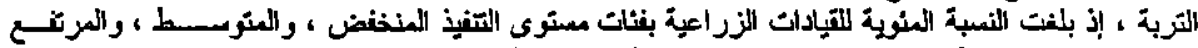

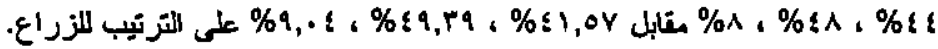

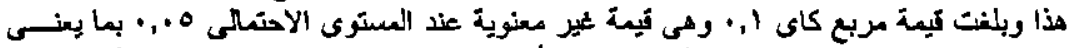

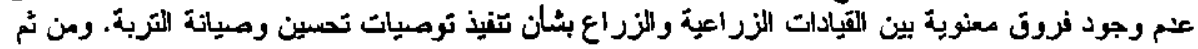

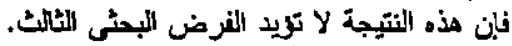

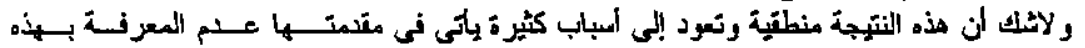

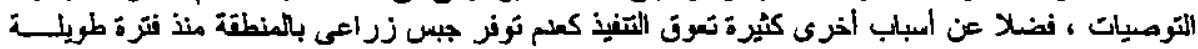

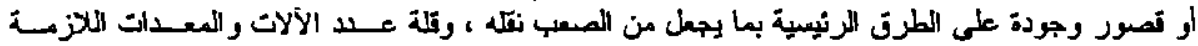
لتطهير التُرع و المصارن والحرث تحت التربة وارتناع تكلفة استجار ها لكثرة الطلب عليها.

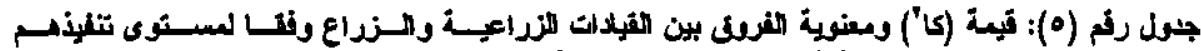

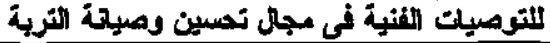

\begin{tabular}{|c|c|c|c|c|c|c|c|}
\hline \multirow{2}{*}{ المَيمة كا' } & \multirow{2}{*}{ الإجملى } & \multicolumn{2}{|c|}{ الزيراع } & \multicolumn{2}{|c|}{ التيّادات الزيراعية } & \multirow{2}{*}{\multicolumn{2}{|c|}{ مستوى تتفبذ التوصبات الفنبة }} \\
\hline & & $\%$ & عدد & $\%$ & عدد & & \\
\hline \multirow{3}{*}{.1} & का & 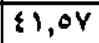 & 19 & $\xi \xi$ & YY & منذفض: ألى من (با ررجة) & \\
\hline & 1.7 & $\varepsilon q, r q$ & Ar & $\llbracket \wedge$ & Y! & متوسط: Y...-Y؛ ررجة) & - \\
\hline & 19 & $9, . \ell$ & 10 & $\Lambda$ & $\varepsilon$ & مرتخع: (بع برجة) فأكثر & - \\
\hline & Y19 & $1 \cdots$ & 19 & T.. & 0. & المجموع & \\
\hline
\end{tabular}

خامسا: دراسة العو امل العرتبطة والمحددة لمستوى تتنيذ كل من القيادات النزاعبة والمنداع للتوصبات

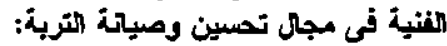

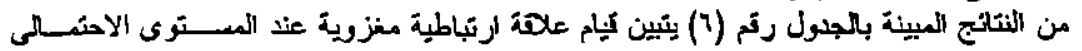

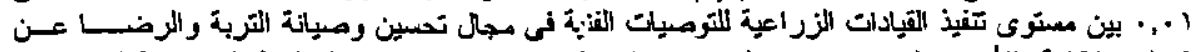

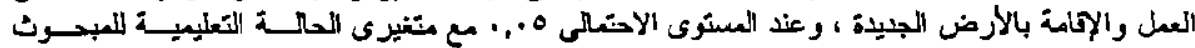

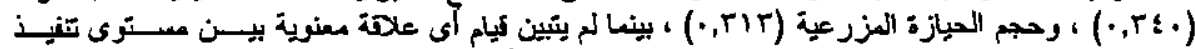

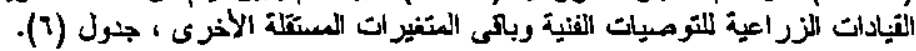




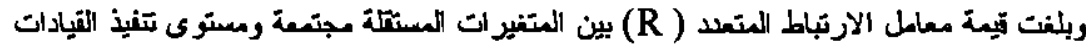

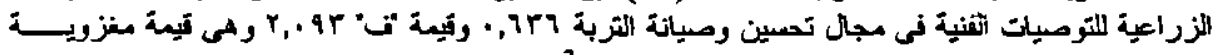

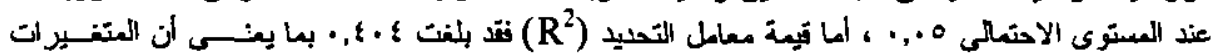

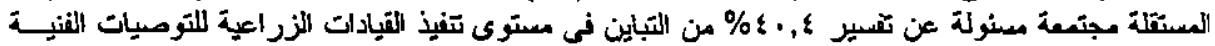

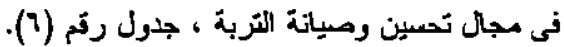

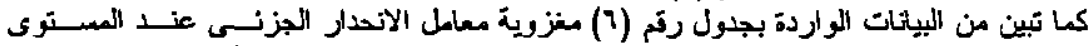

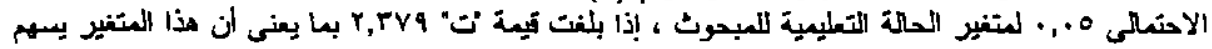

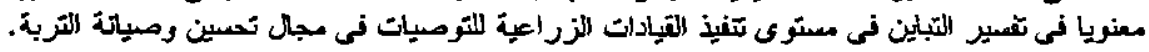

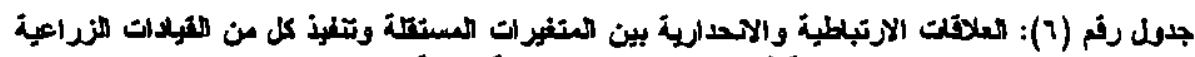

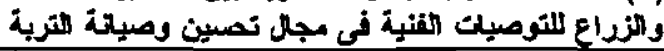

\begin{tabular}{|c|c|c|c|c|c|c|c|c|c|}
\hline & & & & & of & القتبلان & & فنتى الادرلسية & \\
\hline "نه" & الاتصلمل & 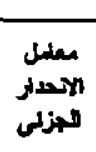 & "الעرتهل & 'تئ & 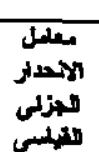 & الاتعلز & الارتهلا معلط & 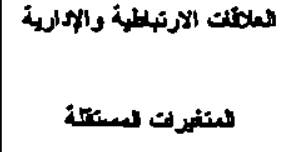 & e \\
\hline $1, r+1$ & $\cdot, 119$ & $Y, 190$ &., $1 \mathrm{rr}$ & $\cdot, 11 \AA$ &.,+14 & $\{, Y 0\}$ & $\cdot . \cdot V Y$ & عمر المبحرث & 1 \\
\hline $0 Y, T \leq Y$ &., 110 &., $1 \mathrm{rV}$ & "थ.19 & . r17- &., $.07-$ & r.vi- & $\cdot, 1 T t$ & 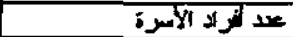 & $T$ \\
\hline$\cdot$, AT. & $0 .+89$ & 7,711 & $\because, 91 \mathrm{~A}$ & Y,rYq & $\cdot, 871$ & $\cdot, \mathrm{TVT}$ & $\because, r \leqslant$. & لحلة التمليمبة للمبحرث & $r$ \\
\hline $1,1: 5-$ & $\cdot,$. ・1- & $0, Y Y,-$ & $\cdot, \cdot 1 \mathrm{Y}-$ & $\cdot, \wedge \cdot\{$ & i,ior & $\cdot, T, P$ & $\because, r \mid r$ & هجم لحبازة لهزراتية & 1 \\
\hline$\because{ }^{\prime}, r Y i$ & $\cdot$. IAV & 0.797 & $\cdot,|11|$ &., $8 T t$ & $\cdot, \cdot 71$ & $9, \cdots 1$ & $\cdot, .1$ & 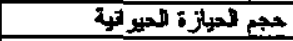 & 0 \\
\hline$\cdot, 11 \mathrm{~V}-$ & $0, .97-$ & $\because 119-$ & $\therefore, .11$ & $\because, 91-$ & $\because \cdot 1 \gamma-$ & $1,11-$ & $\cdot .1 T A$ & 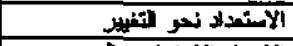 & 7 \\
\hline $1,8 \mathrm{rg}$ & $., 1,0$ & A,rYA & $\because, 1 \times 7$ & $\cdot,\{\leqslant\}$ &..$\cdot 71$ & $8,9.0$ & $\cdot, \cdot \llbracket t r$ & الإسهام الاجتساءم لر سمي & $r$ \\
\hline$\cdot, r T 9$ & $\cdot . Y Y$ & $Y, \cdot \& 1$ & $\cdot .911$ & $1, \cdot$ YA- & $\cdot, Y 0,-$ & $A_{1} \cdot 9-$ & $\cdot, 1 T 9$ & 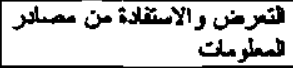 & A \\
\hline$\cdot, r Y .-$ & $\because$ •.ท- & -,IT.- & $\therefore, .10$ & $\cdot 1 \cdot 1-$ & $\cdot .18 .-$ & •,tYA- &. .79 & 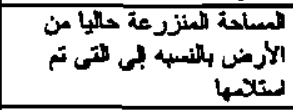 & 1 \\
\hline •, rרq &.,$\times 9$ & $1,09$. & . & 1,609 & $\cdot, T \cdot 9$ & $Y, 009$ & $\cdot, 110$ & 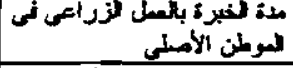 & . \\
\hline $0,9 \mathrm{VI}$ &., $1 \mathrm{VO}$ & A,TAA &., $17 \varepsilon$ & $\cdot .57 !$ & $\because .10$ & $0,10 \mathrm{r}$ &.,$Y Y_{0}$ & الآتجاء نحر الأرثشاد الزراعى & 11 \\
\hline - & $\because$. or & $r, \cdot 1-$ & $0 ., P 7$ & $1, A Y$ & . ror & , rol & $\cdots,, \& \wedge 1$ & 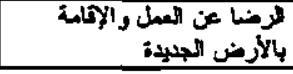 & ir \\
\hline & 7 & 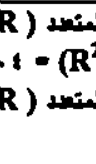 & 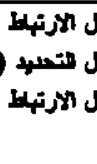 & هية هية & & & & 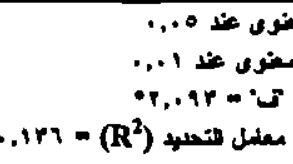 & 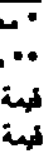 \\
\hline
\end{tabular}

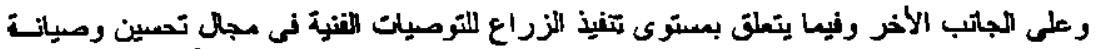

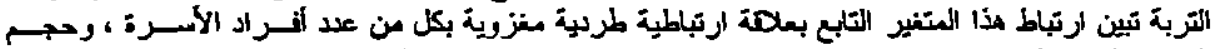

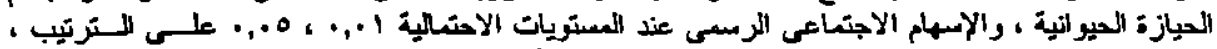

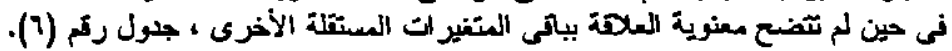

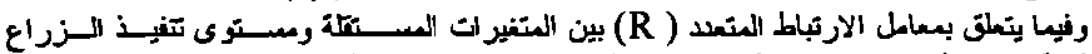

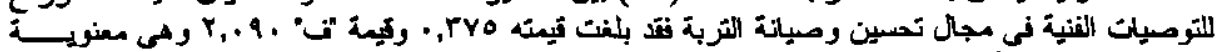

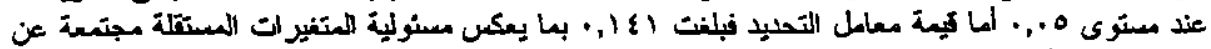

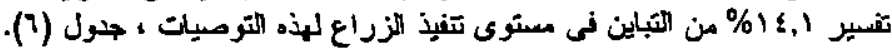

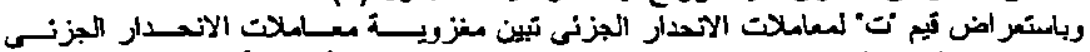

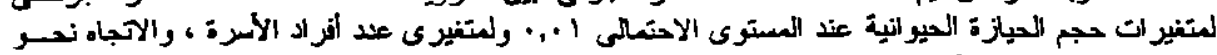

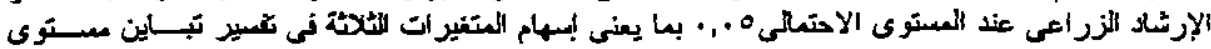

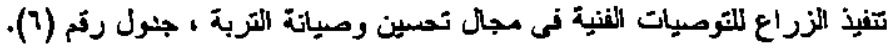




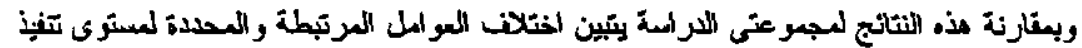
كل منهم اللتوصيات الفنية في مجال تحسين وصيانة التربة. المراجع|بs

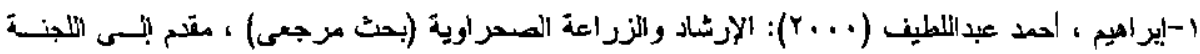

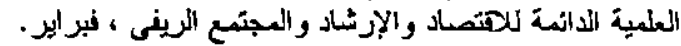

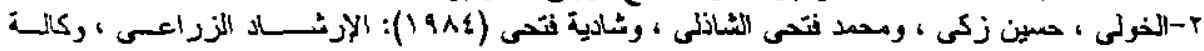
الصتر للمحافة و النثر ، الإسكندرية.

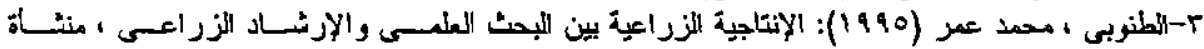
المعارن ، الإسكندرية.

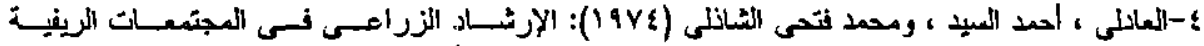

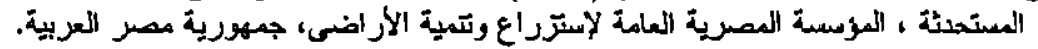

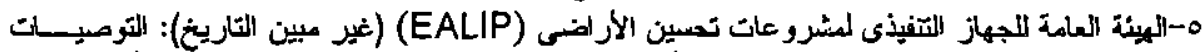

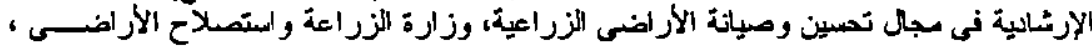

القاهرة.

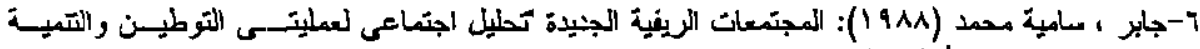

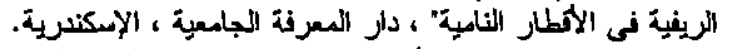

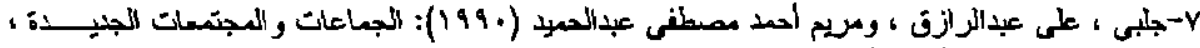
دار المعرفة الجامعية ، الإسكنرية.

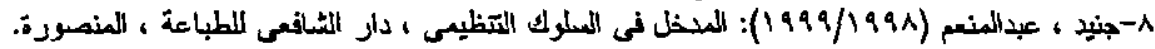

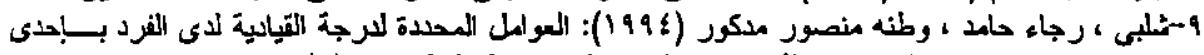

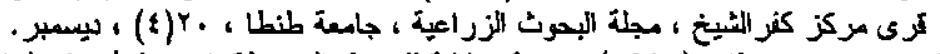

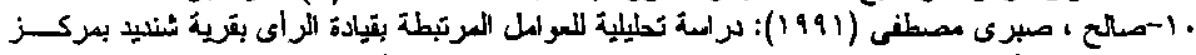

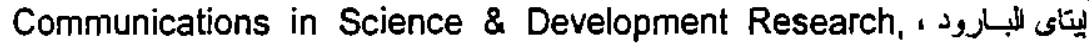
1991, Jan-March, vol.33.

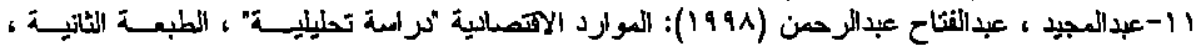

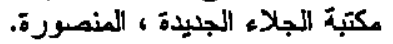

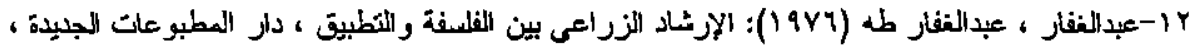
الإسكندرية.

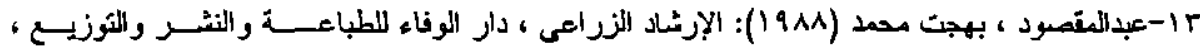
المنصورة.

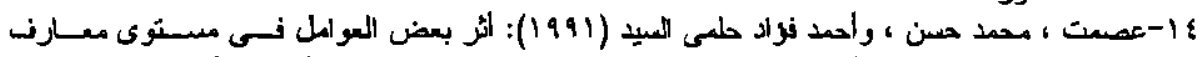

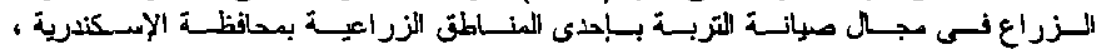
Communications in Science and Development Research, 1991, JanMarch, vol.33.

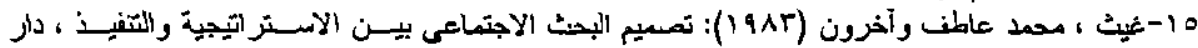
المعرفة الجامعية ، الإسكنرية.

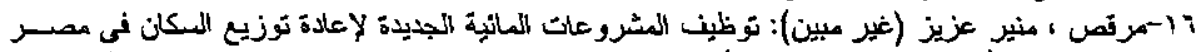

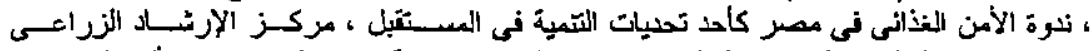

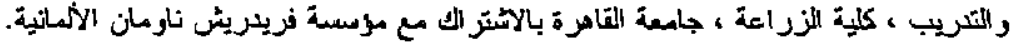

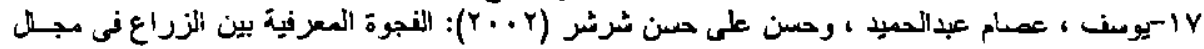

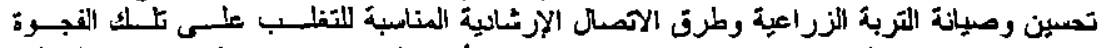

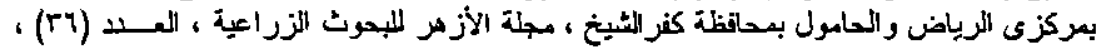

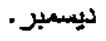




\title{
A COMPARATIVE STUDY BETWEEN AGRICULTURAL LEADERS AND FARMERS IN THE FIELD OF ADVANCEMENT AND MAINTENANCE OF RECLAIMED LAND AT KAFR EL-SHAIKH GOVERNORATE \\ Shalaby, Ebtesam H. and E.S. Mikhael \\ Agric. Ext. and Rural development Research Institute
}

\begin{abstract}
The main objective of this study was to know the differences in the characteristics between Agricultural Leaders and Farmers, and to recognizing the difference in the knowledge level and in the adoption level between Agricultural Leaders and Farmers in the field of advancement and iftintenance of reclaimed land, also to identify the most vital factors that delermine the knowledge level and adoption level of agricultural leaders and farmes.

A well prepared and pretested questionnaire was used to collect data for this 'research, through personal interviews from a vanadium sample consisted of 216 (50 leaders, 166 farmers) in El Taamim, El Taftiesh, El Selam, in Kafer El-Sheikh Governorate.

Percentages, simple correlation coefficient and multiple regression analysis was used to analyze the data statistically.

The most important findings of this study was as follow:

- No significant differences were found among Agri. Leaders and Farmers conceming the knowledge level, and the adoption level.

- There was a significant statistic relationship between all the independent variables of this study and the knowledge of Agri. Leaders, this variables explained about $41.3 \%$ of the variance in the level of Agri Leaders knowledge.

- There was a significant statistical relationship between all the independent variables of this study and the knowledge level of farmers, this variables explained about $13.6 \%$ of the variance in the level of farmers knowledge.

- There was significant statistical relationship between all the independent variables of this study and the adoption of the Agri. cultural leader, this variables explained about $40.4 \%$ of the variance in the level of agricultural leaders adoption.

- There was a significant statistical relationship between all the independent variables of this study and the adoption level of farmers, this variables explained about $14.1 \%$ of the variance in the level of farmers knowledge.
\end{abstract}

